\title{
Adaptación y análisis psicométrico del cuestionario de actitudes sobre diabetes mellitus (DAS-3sp) en Venezuela
}

\section{Adaptation and psychometric analysis of an attitude survey on diabetes mellitus (DAS-3sp) in Venezuela}

\author{
Enmanuel Velásquez-Zambrano ${ }^{1 *}$, Freddy Contreras-Santos ${ }^{1}$ y Luisa Angelucci-Bastidas ${ }^{2,3}$
}

${ }^{1}$ Facultad de Medicina, Escuela de Enfermería, Departamento de Ciencias Básicas, Universidad Central de Venezuela; ${ }^{2}$ Centro de Investigación y Evaluación Institucional, Universidad Católica Andrés Bello; ${ }^{3}$ Departamento de Ciencia y Tecnología del Comportamiento, Universidad Simón Bolívar. Caracas, Venezuela

\section{RESUMEN}

Objetivo: Determinar la fiabilidad, validez de contenido y estructura factorial como indicador de validez de constructo de la versión española de la Diabetes Attitude Scale (DAS-sp3) elaborada por Hernández Anguera, et al. Material y métodos: Estudio psicométrico, donde participaron 100 sujetos con diabetes mellitus tipo 1 y 2, entre 12 y 70 años de edad; el 39\% hombres y el $61 \%$ mujeres que acudían a la consulta de endocrinología en sus respectivos hospitales, que estaban accesibles y que aceptaron participar de manera voluntaria como parte de la muestra. La información se presentó de manera descriptiva, se obtuvieron indicadores de fiabilidad por medio del coeficiente alfa de Cronbach, para la validez de constructo se realizó análisis factorial con el método de componentes principales. Resultados: Se obtuvo un valor total de alfa de Cronbach de 0.62. Los 33 ítems se agruparon en una estructura factorial de seis dimensiones, que explican un $50.32 \%$ de la varianza total. Conclusión: La versión adaptada del DAS-3 ha demostrado satisfactorias cualidades psicométricas, por lo que puede ser aplicada en población venezolana.

Palabras clave: Diabetes. Educación. Análisis factorial. Actitudes. Fiabilidad. Validez.

\begin{abstract}
Objective: To determine the reliability, content validity and factorial structure as an indicator of construct validity of the Spanish version of the Diabetes Attitude Scale (DAS-3) by Hernández Anguera, et al. Material and methods: A psychometric study was performed in 100 subjects with type 1 and 2 diabetes, between $12-70$ years old, 39\% men and 61\% women, who attended the endocrinology consultation in their respective hospitals, who were accessible and who agreed to participate voluntarily as part of the sample. The results were presented descriptively, reliability indicators were obtained through the Cronbach's alpha coefficient, and for the construct validity factorial analysis was performed with the principal component method. Results: A Cronbach's alpha value of 0.62 was obtained. The 33 items were grouped into a 6 dimensions factorial structure, which explain $50.32 \%$ of the total variance. Conclusion: The adapted version of the questionnaire DAS-sp3 has demonstrated satisfactory psychometric qualities and can be applied in subsequent research in the Venezuelan population.
\end{abstract}

Key words: Diabetes. Education. Factor analysis. Attitudes. Reliability. Validity.
Correspondencia:

*Enmanuel Velásquez-Zambrano E-mail: enmanuelvzQgmail.com
Fecha de recepción: 27-07-2020

Fecha de aceptación: 05-09-2020 DOI: 10.24875/ALAD.20000024
Disponible en internet: 16-11-2020 Rev ALAD. 2020;10:143-53

2248-6518 / @ 2020 Asociación Latinoamericana de Diabetes. Publicado por Permanyer. Este es un artículo open access bajo la licencia CC BY-NC-ND (http://creativecommons.org/licenses/by-nc-nd/4.0/). 


\section{INTRODUCCIÓN}

La prevalencia en aumento de distintas enfermedades crónicas ha generado una mayor apreciación de la relación entre lo emocional, lo cognitivo y lo conductual en los procesos de salud y enfermedad. Al evidenciarse que otros factores como la calidad de vida, la conducta y la percepción son tan importantes como los resultados fisiológicos, la necesidad de entender estos procesos conductuales y psicosociales es evidente ${ }^{1}$.

Específicamente, con relación a la diabetes, investigaciones como las de Díaz, et al. ${ }^{2}$ y Bazata, et al. ${ }^{3}$ señalan que pese a conocer las pautas de tratamiento, la mayoría de las personas con diabetes tiene dificultades para seguirlas ininterrumpidamente (en especial el ejercicio físico, el autoanálisis de glucosa y el cumplimiento de la dieta), por lo que los pacientes no trasladan sus conocimientos a conductas.

Las metas de tratamiento podrían verse influenciadas por las motivaciones y necesidades personales, factores sociodemográficos, el ambiente familiar, las condiciones sociolaborales y las actitudes del personal médico, entre otros; por esta causa, es importante analizar los factores personales y psicosociales del paciente si se desea tener éxito en la aplicación del tratamiento ${ }^{1,4}$.

En este sentido, la Asociación Americana de Diabetes (ADA) incluyó en el año 2016 en una edición especial la primera declaración de posición para el cuidado psicosocial de la diabetes, centrada en el paciente ${ }^{5}$. Todo ello orientado hacia un objetivo común, combinar pautas clínicas y educacionales que permitan mejorar los resultados de salud y la calidad de vida de los pacientes ${ }^{6}$.

Recientemente, en 2020 la Asociación Americana de Educadores en Diabetes (AADE) elaboró un modelo basado en la evidencia para la evaluación e intervención de individuos y poblaciones que viven con diabetes y otras enfermedades cardiometabólicas. Este grupo de trabajo identificó siete conductas de autocuidado que promueven el éxito y el autocontrol efectivo de la diabetes conocido como AADE7 (American Association of Diabetes Educator 7). Estas conductas son: afrontamiento saludable, alimentación saludable, estando activo, tomando medicación, supervisión, reducir el riesgo y resolución de problemas. Este marco cambió el enfoque de la educación. Una efectiva educación necesita ir más allá de transferir conocimiento, necesita abordar y apoyar el cambio en el comportamiento para centrarse en la calidad de vida y afectar los resultados clínicos relacionados con la salud. La primera conducta, afrontamiento saludable, definida como una actitud positiva hacia la diabetes y el autocuidado, relaciones positivas con los demás y calidad de vida, es fundamental para el dominio de los otros seis comportamientos. Factores psicosociales que interfieren con la capacidad de una persona para autocontrolar la enfermedad y lograr resultados metabólicos deseados influyen en gran medida en la diabetes y en otras enfermedades relacionadas ${ }^{7}$. Niveles más altos de emociones positivas, mayor apoyo social y actitudes hacia el autocuidado están asociados con un mejor cuidado personal en la diabetes'.

En consecuencia, surge la importancia del aspecto emocional vinculado a la actitud ${ }^{8}$, entendiendo por actitud la predisposición para la adopción de acciones de autocuidado. En el caso particular del manejo de la diabetes, esa predisposición propicia la reducción del estrés asociado a la enfermedad, una mayor receptividad al tratamiento, la confianza en el equipo de salud, mejora la autoestima, la percepción más positiva acerca de la salud y la aceptación social9.

Por ello es aconsejable contar con un instrumento validado que posibilite la obtención de información sobre las actitudes de los pacientes. 
Dentro de los cuestionarios conocidos para medir las actitudes en diabetes se encuentran el Health Knowledge, Attitudes and Practices Questionnaire ${ }^{10}$, el Diabetes Attitudes, Wishes and Needs 2 (DAWN) ${ }^{11}$ y uno de los más utilizado, la Diabetes Attitude Scale (DAS-3), que fue elaborada por Anderson, et al. ${ }^{12}$ entre los años 1995 y 1996 con el fin de mejorar un instrumento para evaluar las actitudes y motivaciones relacionadas con diabetes mellitus de pacientes y profesionales sanitarios.

El cuestionario DAS-3 fue traducido y adaptado al idioma castellano en el año 2000 para la población española por Hernández Anguera, et al. ${ }^{13}$. El cuestionario, de 33 preguntas, está compuesto por cinco dimensiones: necesidad de entrenamiento especial, percepción de la gravedad, valoración del control estricto, valoración del impacto psicosocial y autonomía del paciente. Se obtuvo un resultado global de consistencia interna de 0.74 , lo que garantiza la fiabilidad del cuestionario. Se dispone, por tanto, de un instrumento útil para evaluar actitudes y motivaciones que condicionan la efectividad de la intervención educativa y terapéutica.

Dada la utilidad del instrumento en la población española, los investigadores se propusieron determinar la fiabilidad y validez de la versión española de la DAS-3 en población venezolana, adaptándola a las características idiosincráticas y culturales del país.

\section{MATERIAL Y MÉTODOS}

\section{Muestra}

Se seleccionó únicamente a los pacientes que acudieron a control en los servicios de endocrinología de tres hospitales tipo IV según las normas de clasificación de establecimientos de atención médica del subsector salud en Venezuela ${ }^{14}$, que cumplieran los
TABLA 1. Características sociodemográficas de los participantes $(n=100)$

\begin{tabular}{|c|c|c|}
\hline & Frecuencia & Porcentaje \\
\hline \multicolumn{3}{|l|}{ Sexo } \\
\hline Femenino & 61 & 61 \\
\hline Masculino & 39 & 39 \\
\hline \multicolumn{3}{|l|}{ Grado de instrucción } \\
\hline $6 .^{\circ}$ grado & 25 & 25 \\
\hline $9 .^{\circ}$ grado & 9 & 9 \\
\hline Bachiller & 26 & 26 \\
\hline Técnico superior & 12 & 12 \\
\hline Universitario & 28 & 28 \\
\hline \multicolumn{3}{|l|}{ Tipo de diabetes } \\
\hline Diabetes tipo 1 & 33 & \\
\hline Diabetes tipo 2 & 67 & \\
\hline \multicolumn{3}{|l|}{ Grupo etario } \\
\hline $12-20$ años & 15 & 15 \\
\hline $21-40$ & 17 & 17 \\
\hline $41-65$ & 58 & 58 \\
\hline$>65$ & 10 & 10 \\
\hline \multicolumn{3}{|l|}{ Hospital } \\
\hline Universitario de Caracas & 70 & 70 \\
\hline Dr. Carlos Arvelo & 20 & 20 \\
\hline Dr. Miguel Pérez Carreño & 10 & 10 \\
\hline
\end{tabular}

criterios de inclusión: edad comprendida entre 12 y 70 años de edad, diagnóstico establecido de diabetes mellitus tipo 1 y tipo 2 en un tiempo no menor de seis meses, de ambos sexos, con como mínimo 6. grado de instrucción aprobado, que recibían tratamiento con insulina o con medicamentos orales asociados o no a insulina, que estuvieran accesibles y que aceptaran participar de manera voluntaria como parte de la muestra (Tabla 1). Los hospitales seleccionados fueron: Hospital Universitario de Caracas (HUC), Hospital Dr. Carlos Arvelo y Hospital Dr. Miguel Pérez Carreño, que funcionan como centros de referencia nacional y son los de mayor 
TABLA 2. Ecuación de corrección para cada dimensión del Diabetes Attitude Scale (DAS-3)

\begin{tabular}{|c|c|c|}
\hline Dimensión & Ecuación de corrección & Instrucciones \\
\hline Necesidad de entrenamiento especial & $\Sigma(1,6,10,17,20) / n .^{\circ}$ de preguntas contestadas & \\
\hline Percepción de la gravedad & $\begin{array}{l}\Sigma(2,7,11,15,21,25,31) / \mathrm{n} .^{\circ} \text { de preguntas } \\
\text { contestadas }\end{array}$ & Valores inversos para $\mathrm{P} 2,7,11$ y 15 \\
\hline Valoración del control estricto & $\begin{array}{l}\Sigma(3,8,12,16,23,26,28) / n .^{\circ} \text { de preguntas } \\
\text { contestadas }\end{array}$ & Valores inversos para $\mathrm{P}_{3}, 16,23,26$ y 28 \\
\hline Valoración del impacto psicosocial de la DM & $\begin{array}{l}\Sigma(4,13,18,22,29,33) / n .^{\circ} \text { de preguntas } \\
\text { contestadas }\end{array}$ & Valores inversos para $\mathrm{P} 13$ \\
\hline Autonomía del paciente & $\begin{array}{l}\Sigma(5,9,14,19,24,27,30,32) / n .^{\circ} \text { de preguntas } \\
\text { contestadas }\end{array}$ & \\
\hline
\end{tabular}

DM: diabetes mellitus.

tamaño del país, lo que confiere una mayor representatividad a la muestra. El estudio se efectuó entre los meses de julio y septiembre de 2015.

Se consideró adecuado incluir 100 sujetos para determinar la fiabilidad y análisis factorial, dada la recomendación para el cálculo del tamaño de la muestra en estudios factoriales: incluir 100 pacientes o cinco sujetos, como mínimo, por cada ítem que compone el cuestionario ${ }^{15}$. El proyecto inicial tuvo la aprobación del comité de bioética del HUC.

\section{Tipo de investigación}

Investigación de enfoque instrumental psicométrico, donde se obtienen indicadores de fiabilidad y validez de una escala de medición, tipo autorreporte.

\section{Instrumento}

El cuestionario usado para la recolección de los datos correspondió a la versión española de la DAS-3 traducida al castellano, adaptada y validada por Hernández Anguera, et al. ${ }^{13}$.
El cuestionario de 33 preguntas está compuesto por cinco dimensiones: 1 ) necesidad de entrenamiento especial $(1,6,10,17,20), 2)$ percepción de la gravedad $(2,7,11,15,21,25,31), 3)$ valoración del control estricto $(3,8,12,16,23,26,28), 4)$ valoración del impacto psicosocial de la $\operatorname{DM}(4,13,18,22,29,33)$ y 5) autonomía del paciente $(5,9,14,19,24,27,30$, 32). Para cada pregunta la puntuación máxima es 4 y la mínima 1, según el sujeto responda: «totalmente de acuerdo» (4 puntos), «de acuerdo» (3 puntos), «en desacuerdo» (2 puntos) o «totalmente en desacuerdo» (1 punto). En la tabla 2 se muestra la ecuación de corrección que se utiliza para obtener la puntuación de cada dimensión.

\section{Procedimiento}

En la presente investigación para la adaptación del cuestionario se realizó una validación de contenido que se determinó por medio de la fundamentación teórica de cada uno de los ítems en relación con el constructo medido ${ }^{16,17}$. Considerando que los ítems eran suficientemente claros solo se hicieron pequeñas modificaciones a las preguntas con la única finalidad de hacerlas más cortas y sin que estas desviaran su objetivo a lo que se pretendía conocer, de manera que fueran fácilmente comprensibles y no 
requirieran ninguna otra explicación. Se eliminó la palabra «diabético» para evitar sentimientos de discriminación y estigmatizantes.

En cuanto a las respuestas, de las cinco opciones del cuestionario de Hernández Anguera, et al. ${ }^{13}$ (totalmente de acuerdo, de acuerdo, indiferente, en desacuerdo, totalmente en desacuerdo) se eliminó la opción «indiferente» para eludir la tendencia de marcar el punto medio y evitar el sesgo por indecisión ${ }^{18,19}$.

Luego de realizar estos cambios al cuestionario, se aplicó a una muestra piloto de 50 personas para determinar el tiempo que tomaba realizarla (15-20 minutos) y si existía una buena comprensión de los ítems.

Previa lectura, comprensión y firma del consentimiento informado, se aplicó el cuestionario a la muestra seleccionada de manera autoadministrada; los participantes marcaron respecto a cada información la categoría que mejor describía su reacción o respuesta.

Luego de la recolección de los datos se determinó la fiabilidad y validez. Se obtuvo el indicador de fiabilidad abordando el criterio de homogeneidad, mediante el coeficiente de alfa de Cronbach, tanto de la escala completa como para cada una de sus dimensiones, estableciendo como valor confiable un alfa de Cronbach mayor a o.6.

La validez de constructo se comprobó por medio del análisis factorial con rotación tipo varimax, dado que se asume la ortogonalidad entre las dimensiones, es decir, que no hay correlación o es muy baja entre ellas. Para considerar a un ítem dentro de una dimensión se tomó como límite inferior la carga factorial de 0.350. Luego de determinar las nuevas dimensiones se obtuvo la fiabilidad de cada una.
TABla 3. Coeficientes de fiabilidad por dimensión y forma total de la encuesta

\begin{tabular}{|l|c|}
\hline Dimensiones & Alfa de Cronbach \\
\hline Necesidad de entrenamiento especial & 0.67 \\
\hline Percepción de la gravedad & 0.48 \\
\hline Valoración del control estricto & 0.99 \\
\hline Valoración del impacto psicosocial & 0.97 \\
\hline Autonomía del paciente & 0.99 \\
\hline Total & 0.62 \\
\hline
\end{tabular}

\section{RESULTADOS}

Se incluyeron 100 sujetos, de los cuales el 61\% eran mujeres y el $39 \%$ hombres, en su mayoría (67\%) con diabetes tipo 2, con edad entre 41 y 65 años; el $28 \%$ de estos pacientes tenía un tratamiento combinado de fármacos orales e insulina, en contraste con el $33 \%$ de sujetos con diabetes tipo 1, más jóvenes (entre 12 y 40 años de edad). En cuanto al nivel académico, un $60 \%$ manifestó tener estudios de bachillerato completo o incompleto, el restante $40 \%$ afirmó tener algún tipo de estudio universitario.

Los resultados obtenidos en el cálculo del coeficiente de alfa de Cronbach en la forma total de la encuesta y según las cinco áreas evaluadas han mostrado una consistencia interna buena, con coeficientes entre 0.66 y $0.99^{20,21}$, con excepción de la subescala que hace referencia a la «percepción de la gravedad», que presentó una baja o nula fiabilidad (Tabla 3).

Como acercamiento a la validez de constructo se evaluó la estructura factorial de la encuesta mediante el método de componentes principales. Al utilizar como criterios valores propios con valores propios (Eigenvalues) mayores a 2 (Fig. 1) y una varianza acumulada de por lo menos un $50 \%$, se obtuvieron seis factores que explican en conjunto un $50.32 \%$ de la varianza total. 


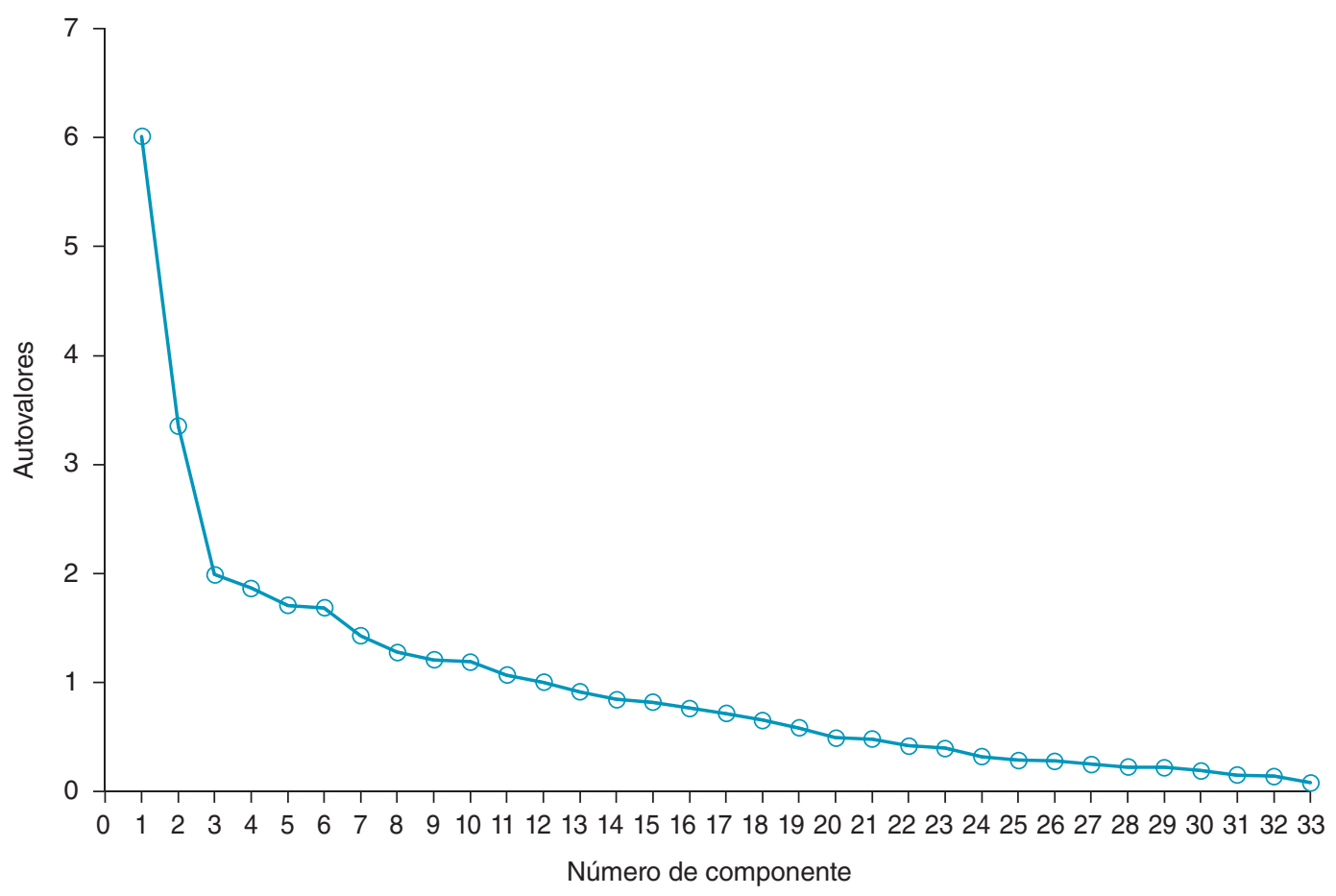

Figura 1. Gráfico de sedimentación para los 33 ítems del cuestionario de actitudes sobre la diabetes mellitus.

Mediante una rotación varimax y tomando como criterio una carga factorial mayor a 0.350 , la estructura de seis factores fue como se muestra en la tabla 4:

- Dimensión 1, «necesidad de entrenamiento especial». Incorporó el $12.453 \%$ de la varianza total e incluyó los siguientes ítems: 6, 7, 9, 10, 12, 17, 19 y 20.

- Dimensión 2, «control estricto». Incorporó el $10.847 \%$ de la varianza total e incluyó los siguientes ítems: 1, 3, 15, 16, 23, 26, 28, 29 y 31.

- Dimensión 3, «impacto psicosocial». Incorporó el $8.494 \%$ de la varianza total e incluyó los siguientes ítems: 4,18, 21 y 22.

- Dimensión 4, «apoyo familiar» (nueva dimensión). Incorporó el $6.543 \%$ de la varianza total e incluyó los siguientes ítems: 8, 24 y 33.
- Dimensión 5, «autonomía del paciente». Incorporó el $6.216 \%$ de la varianza total e incluyó los siguientes ítems: 5, 14, 30 y 32 .

- Dimensión 6, «percepción de la gravedad». Incorporó el $5.774 \%$ de la varianza total e incluyó los siguientes ítems: 2, 25 y 27.

Los ítems con carga negativa y los que no cargaron en algún factor $(11[-0,447]$ y 13 [0]) fueron eliminados. Lo que permite proponer un instrumento de 31 ítems (Tabla 5).

Luego de determinar las dimensiones se obtuvieron los coeficientes de alfa de Cronbach para cada una de ellas con un valor entre 0.72 y 1.0, con la excepción de la dimensión «percepción de la gravedad», con 0.24 (Tabla 6).

En líneas generales se obtiene una fiabilidad satisfactoria, que indica consistencia interna y una estructura factorial coherente. 
TABLA 4. Matriz de componentes rotados. Componentes

\begin{tabular}{|c|c|c|c|c|c|c|}
\hline Ítems & 1 & 2 & 3 & 4 & 5 & 6 \\
\hline 20 & 0.771 & & & & & \\
\hline 12 & 0.700 & & & & & \\
\hline 9 & 0.698 & & & & & \\
\hline 10 & 0.681 & & & & & \\
\hline 6 & 0.605 & & & & & \\
\hline 17 & 0.595 & & & & & \\
\hline 28 & & 0.828 & & & & \\
\hline 15 & & 0.703 & & & & \\
\hline 23 & & 0.690 & & & & \\
\hline 26 & & 0.569 & & & & \\
\hline 29 & & 0.527 & & & & \\
\hline 16 & & 0.501 & & & & 0.373 \\
\hline 3 & & 0.468 & & & & \\
\hline 1 & & 0.466 & & & & \\
\hline 31 & & 0.366 & & & & \\
\hline 21 & & & 0.757 & & & \\
\hline 22 & & & 0.701 & & & \\
\hline 19 & 0.380 & & -0.563 & & & \\
\hline 18 & & & 0.559 & & & \\
\hline 4 & & & 0.523 & & & \\
\hline 13 & & & & & & \\
\hline 8 & & & & 0.711 & & \\
\hline 33 & & & & 0.686 & & \\
\hline 11 & & & & -0.447 & & \\
\hline 24 & & & & 0.433 & & \\
\hline 14 & & & & & 0.738 & \\
\hline 30 & & & & & 0.589 & \\
\hline 5 & & & & & 0.462 & \\
\hline 7 & 0.422 & & & -0.371 & -0.450 & \\
\hline 32 & & & & & 0.351 & \\
\hline 27 & & & & & & 0.615 \\
\hline 25 & & & -0.437 & & -0.363 & 0.609 \\
\hline 2 & & & & & & 0.523 \\
\hline
\end{tabular}

\section{DISCUSIÓN}

Una actitud positiva favorece la valoración de la enfermedad como desafío, lo que promueve la aparición de estrategias activas de afrontamiento, más apropiadas para mejorar el cumplimiento de las pautas de autocuidado y prevenir las complicaciones a corto y largo plazo ${ }^{8}$.

Para medir la actitud, el presente estudio adaptó a la población venezolana la versión española de la DAS-3 de Hernández Anguera, et al. ${ }^{13}$, para posteriormente realizar un análisis psicométrico de esta.

El alfa de Cronbach global del cuestionario (0.62) puede considerarse como buena tomando en cuenta que se utilizó para fines de investigación teóri$\mathrm{Ca}^{20,21}$, además es equiparable con el estudio de Hernández Anguera, et al. ${ }^{13}$, quienes consiguieron un valor similar en el coeficiente (0.66) en pacientes con diabetes. Cabe destacar que cuando Hernández Anguera, et al. ${ }^{13}$ incluyeron en su muestra a profesionales sanitarios (médicos y enfermeras) y pacientes el coeficiente arrojó un resultado global de consistencia interna de 0.74 , lo que indica que al considerarse solo los pacientes la consistencia interna entre los ítems disminuye.

En cuanto a la fiabilidad por dimensiones y específicamente a la que refiere «percepción de la gravedad» fue de 0.48 vs. 0.50 de pacientes encuestados por Hernández Anguera, et al. ${ }^{13}$, pero por debajo de los resultados de la encuesta original elaborada por Anderson, et al. ${ }^{12}$, que obtuvo 0.80 de fiabilidad para esta dimensión. Esto podría explicarse por las diferencias atribuibles a aspectos culturales entre la población estadounidense y la de la presente investigación en cuanto a la percepción de la gravedad. Se ha encontrado que las percepciones y las clasificaciones sociales e individuales sobre el proceso salud enfermedad están determinadas por el contexto 
TABla 5. Adaptación del cuestionario de actitudes sobre diabetes mellitus en Venezuela

Generalmente (yo) creo que: para comunicarse.

2. La gente que NO necesita insulina para su tratamiento es porque está mejor (menos grave).

3. No tiene sentido tratar de tener un buen control de azúcar en la sangre porque de todas formas aparecerán complicaciones.

4. La diabetes afecta casi todos los aspectos de la vida.

5. Las decisiones importantes del cuidado diario deberían tomarse por la misma persona.

6. Los profesionales de la salud deberían ser enseñados (entrenados) sobre cómo el cuidado diario afecta a la vida de los pacientes.

7. La gente mayor con diabetes tipo 2 no suele tener complicaciones.

8. Mantener el nivel de azúcar próximo al nivel normal puede prevenir las complicaciones.

9. Los profesionales de la salud deberían ayudar a sus pacientes a tomar decisiones razonadas sobre sus planes de salud.

10. Es importante que enfermeras y nutricionistas educadores en diabetes aprendan técnicas de comunicación y educación terapéutica.

11. Todas las personas deberían hacer lo que fuera para mantener su nivel de azúcar próximo (cerca) a la normalidad.

12. Las personas deberían tener la última decisión (palabra) sobre sus objetivos de azúcar en sangre.

13. No es necesario pinchar el dedo para medir el azúcar en personas que tienen diabetes tipo 2.

14. Los problemas causados por niveles bajos de azúcar en sangre (hipoglucemia) hacen que un control estricto sea demasiado arriesgado.

15. Los profesionales de la salud deberían aprender a fijar objetivos en colaboración con sus pacientes, no solo decirles qué deben hacer.

16. La diabetes es «agotadora» porque nunca puedes despreocuparte de ella.

17. La persona con diabetes es el elemento más importante del equipo de cuidado.

18. Para hacer un buen trabajo los educadores en diabetes deberían aprender sobre lo que significa ser un profesor. Para ser eficaces los educadores han de tener un elevado grado de conocimientos sobre el proceso de la enseñanza y el aprendizaje.

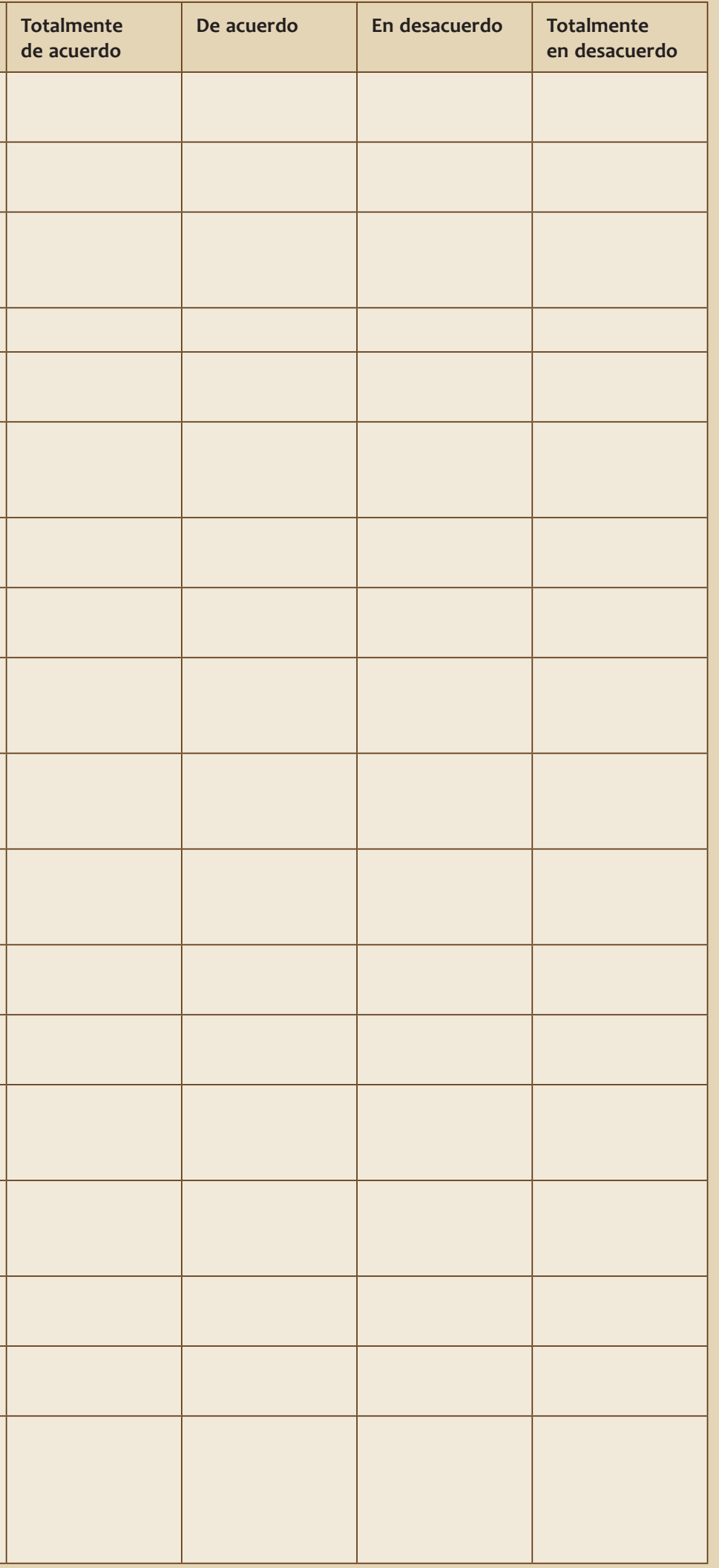


TABLA 5. Adaptación del cuestionario de actitudes sobre diabetes mellitus en Venezuela (continuación)

\begin{tabular}{|c|c|c|c|c|}
\hline Generalmente (yo) creo que: & $\begin{array}{l}\text { Totalmente } \\
\text { de acuerdo }\end{array}$ & De acuerdo & En desacuerdo & $\begin{array}{l}\text { Totalmente } \\
\text { en desacuerdo }\end{array}$ \\
\hline \multicolumn{5}{|l|}{ 19. La diabetes tipo 2 es una enfermedad muy seria (grave). } \\
\hline \multicolumn{5}{|l|}{$\begin{array}{l}\text { 20. El hecho de tener diabetes cambia la perspectiva de vida } \\
\text { de la persona. }\end{array}$} \\
\hline \multicolumn{5}{|l|}{$\begin{array}{l}\text { 21. Probablemente no obtendrás mayor beneficio de un } \\
\text { estricto control de tus niveles de azúcar en sangre. }\end{array}$} \\
\hline \multicolumn{5}{|l|}{$\begin{array}{l}\text { 22. Deberías estar bien informado para poder ocuparte } \\
\text { de tu control. }\end{array}$} \\
\hline \multicolumn{5}{|l|}{ 23. La diabetes tipo 2 es tan grave como la diabetes tipo 1.} \\
\hline \multicolumn{5}{|l|}{ 24. Un control excesivo (estricto) es demasiado trabajo. } \\
\hline \multicolumn{5}{|l|}{$\begin{array}{l}\text { 25. Lo que tú hagas tiene más impacto sobre el resultado } \\
\text { del cuidado de la diabetes que cualquier cosa que haga } \\
\text { un profesional de la salud. }\end{array}$} \\
\hline \multicolumn{5}{|l|}{$\begin{array}{l}\text { 26. Un control estricto de azúcar en sangre solo tiene sentido } \\
\text { para los que tienen diabetes tipo } 1 .\end{array}$} \\
\hline \multicolumn{5}{|l|}{ 27. Es frustrante ocuparte de tu enfermedad. } \\
\hline \multicolumn{5}{|l|}{$\begin{array}{l}\text { 28. Tengo derecho a decidir la rigurosidad con que he de } \\
\text { esforzarme para controlar el nivel de azúcar en sangre. }\end{array}$} \\
\hline \multicolumn{5}{|l|}{$\begin{array}{l}\text { 29. Las personas que toman pastillas deben de preocuparse } \\
\text { por su nivel de azúcar en sangre tanto como aquellas } \\
\text { que utilizan insulina. }\end{array}$} \\
\hline \multicolumn{5}{|l|}{$\begin{array}{l}\text { 30. Las personas con diabetes tienen derecho a NO cuidarse } \\
\text { de su enfermedad. }\end{array}$} \\
\hline $\begin{array}{l}\text { 31. El apoyo de la familia y de los amigos es importante } \\
\text { en el tratamiento. }\end{array}$ & & & & \\
\hline
\end{tabular}

TABLA 6. Coeficientes de fiabilidad de las nuevas dimensiones

\begin{tabular}{|l|c|}
\hline Dimensiones & Alfa de Cronbach \\
\hline Necesidad de entrenamiento especial & 1.00 \\
\hline Control estricto & 0.724 \\
\hline Impacto psicosocial & 0.998 \\
\hline Apoyo familiar & 1.00 \\
\hline Autonomía del paciente & 0.986 \\
\hline Percepción de la gravedad & 0.248 \\
\hline
\end{tabular}

cultural. Estas percepciones logran incorporarse en las creencias populares, las cuales funcionan como esquemas cognitivos, estereotipados, que guían el comportamiento de las personas ${ }^{22}$.
El resto de las subescalas presentan una excelente fiabilidad, lo que sugiere que cada una de ellas mide un constructo unitario y poseen poco error aleatorio, con excepción de la dimensión «percepción de la gravedad», que presentó una fiabilidad baja o nula, por lo que sugerimos revisar todos los ítems o eliminar la subescala, con lo que aumentaría la fiabilidad del cuestionario.

Uno de los aportes de este estudio respecto a la versión española y estadounidense fue que se obtuvo la validez de constructo mediante análisis factorial que indicó la presencia de seis dimensiones, las cuales presentan una configuración de ítems diferentes, en comparación con los estudios de 
Anderson, et al. ${ }^{12}$ y Hernández Anguera, et al. ${ }^{13}$. Los seis factores presentaron un valor propio superior a 2 y explican una varianza del $50.32 \%$, lo que se considera bueno en ciencias sociales, de la salud y del comportamiento. Los dos primeros componentes (necesidad de entrenamiento especial y control estricto) tienden a agrupar un mayor número de variables. El hecho de que un determinado factor sea el que más varianza explique significa que es el elemento que más dispersión mide, es decir, que explica mejor las diferencias entre los distintos individuos de la muestra y por lo tanto es percibido por los respondientes como el más importante. Las cargas son claras, por lo que no existe ambigüedad en la selección de variables por factor ${ }^{23}$. Se incluyó un nuevo factor denominado «apoyo familiar», acorde con la cultura venezolana, donde el componente familiar es importante en la atención de la enfermedad.

Se eliminó la palabra «valoración» de las dimensiones 2 y 3, propuesta en la versión española, por considerarse que al hacer pruebas de autorreporte todo es percepción o valoración, no hay una medición objetiva; además, el término valoración con frecuencia se utiliza para asignar importancia a algo, tal vez más relacionado con la noción de valor, lo que en este caso no tiene justificación, dado que todas las dimensiones miden actitud.

En Venezuela no existe ningún cuestionario validado para medir las actitudes de personas con diabetes, la versión adaptada del cuestionario de actitudes sobre diabetes en idioma castellano del presente estudio ha documentado, por medio de un proceso sistemático, satisfactorias cualidades psicométricas, demostrando ser confiable y válido, y mide el contenido que los proveedores de salud consideran importante para el cuidado del paciente con diabetes, pudiendo ser aplicado en investigaciones posteriores en la población descrita.
La encuesta puede ponerse en práctica utilizando las cinco dimensiones del cuestionario de Hernández Anguera, et al. ${ }^{13}$ o con las dimensiones arrojadas en la presente investigación. Sin embargo, es recomendable seguir realizando estudios de tal manera que pueda ampliarse el alcance del instrumento.

Aunque la muestra tomada se considera suficiente valdría la pena probar el mismo cuestionario en una muestra mucho más grande para tener una mayor validez externa de la investigación. Asimismo, es conveniente obtener otros indicadores de validez, como la convergente, divergente o de criterio, sobre todo esta última, dado que probaría en qué medida el puntaje del instrumento permite predecir la adherencia al tratamiento, comportamiento fundamental en pacientes con diabetes.

\section{FINANCIAMIENTO}

La presente investigación no ha recibido ayudas específicas provenientes de agencias del sector público, sector comercial o entidades sin ánimo de lucro.

\section{CONFLICTO DE INTERESES}

Los autores declaran no tener conflicto de intereses alguno.

\section{RESPONSABILIDADES ÉTICAS}

Protección de personas y animales. Los autores declaran que para esta investigación no se han realizado experimentos en seres humanos ni en animales.

Confidencialidad de los datos. Los autores declaran que han seguido los protocolos de su centro de trabajo sobre la publicación de datos de pacientes. 


\section{Derecho a la privacidad y consentimiento informa- do. Los autores han obtenido el consentimiento in- formado de los pacientes y/o sujetos referidos en el artículo. Este documento obra en poder del autor de correspondencia.}

\section{BIBLIOGRAFÍA}

1. Weinger K, Groot M, Cefalu W. Psychosocial research and care in diabetes: Altering lives by understanding attitudes. Diabetes Care. 2016;26:2122-5.

2. Díaz L, Galán S, Fernández G. Grupo de autocuidado de diabetes mellitus tipo 2. Salud Pública Méx.1993;35(2):169-76.

3. Bazata D, Robinson JG, Fox K, Grandy S. Affecting behavior change in individuals with diabetes. Findings from the Study to Help Improve Early Evaluation and Management of Risk Factors Leading to Diabetes. Diabetes Educ. 2008;34(6):1025-36.

4. Mendoza Rivera R, Gil Alfaro I, Sainz Vázquez L, Soler Huerta E, Mendoza Sánchez H, Hernández Anguera J. Actitudes y motivaciones del paciente diabético y el personal de salud sobre la diabetes tipo 2. Archiv Med Fam. 2009;11(3):93-9.

5. Young-Hyman D, Groot M, Hill-Briggs F, González J, Hood K, Peyrot M. Psychosocial care for people with diabetes: A position statement of the American Diabetes Association. Diabetes Care. 2016;39:2126-40.

6. Martins MC. Diseño y validación de cuestionarios. Matronas Prof. 2004;5(17):23-9.

7. American Association of Diabetes Educator. An effective model of diabetes care and education. Diabetes Educ. 2020;46(2):139-60.

8. Pupko V, Azzollini S. Actitudes, afrontamiento y autocuidado en pacientes con diabetes tipo 2. Rev Argent Salud Pública. 2012;3(10):15-23.

9. Luchetti F, Zanetti M, Dos Santos M, Martins T, Sousa V, Sousa C. Conocimientos y actitudes: Componentes para la educación en diabetes. Rev Latino-Am Enferm. 2009;17(4):28-35.

10. National Health medical Research Concil. AusDiab 2005: The Australian Diabetes, Obesity and Lifestyle study. Tracking the accelerating epidemic: it cause and outcomes. Melbourne: International Diabetes Institute; 2006.

11. Peyrot M, Kovacs K, Davies M, Forbes A, Hermanns $N$, Holt R, et al. Diabetes Attitudes, Wishes and Needs 2 (DAWN2): A multinational, multi stakeholder study of psychosocial issues in diabetes and person - centred diabetes care. Diabetes Res Clin Pract. 2013; 99:174-84.

12. Anderson RM, Funnell MM, Fitzgeral JT, Gruppen LD. The third versión of the diabetes attitude scale. Diabetes Care. 1998;21:1403-7.

13. Hernández Anguera J, Basora Gallisa J, Ansa Echeverría X, Piñol Moreso J, Millán Guasch M, Figuerola Pino D. La versión española de la Diabetes Attitude Scale (DAS 3 sp): un instrumento de medición de actitudes y motivaciones en diabetes. Endocrinol Nutr. 2002;49(9): 293-98.

14. Gaceta Oficial de la República de Venezuela $N^{\circ}$ 32.650. Decreto $\mathrm{N}^{\circ} 1.798 ; 21$ de enero de 1983.

15. Lloret-Segura S, Ferreres-Traver A, Hernández-Baeza A, tomás-Marco I. Análisis factorial exploratorio de los ítems: una guía práctica, revis ada y actualizada. Anal Psicol. 2014;30(3):1151-69.

16. Barraza A. La consulta a expertos como estrategia para la recolección de evidencias de validez basadas en el contenido. Revista de Investigación Educativa. 2007;7:1-14.

17. Sánchez R, Echeverry J. Validación de escalas de medición de salud. Rev Salud Pública. 2004;6(3):302-18.

18. Castro A, Hernández S, Riquelme E, Ossa C, Aedo J, Da Costa S, et al. Nivel de sesgos cognitivos de representatividad y confirmación en estudiantes de psicología de tres universidades del Bio-Bio. Propós Represent. 2019;7(2):210-39.

19. Manterola C, Otzen T. Los sesgos en la investigación clínica. Int J Morphol. 2015;33(3):1156-64.

20. Morales Vallejo P. La fiabilidad de los test y escalas. Estadística aplicada a las ciencias sociales. Madrid, España: Edición Digital; 2008. pp. 3-36.

21. Celina H, Campo A. Aproximación al uso del coeficiente de alfa Cronbach. Rev Colomb Psiquiatr. 2005;34(4):572-80.

22. Quinceno JM, Vinaccia S. Percepción de la enfermedad y características sociodemográficas en enfermos crónicos. Psicol Salud. 2019;29(2): 159-66.

23. Manrique Abril F, Fernández A, Velandia A. Análisis factorial de la Escala Valoración de Agencia de Autocuidado (ASA) en Colombia. Aquichan. 2009;9(3):222-35. 